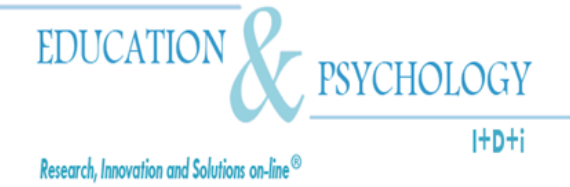

\title{
Programa de enriquecimiento de la creatividad para alumnas sobresalientes de zonas marginadas
}

\section{Blanca Ivet Chávez, Fabiola Zacatelco, Guadalupe Acle}

Facultad de Estudios Superiores Zaragoza,

Universidad Nacional Autónoma de México, Distrito Federal

\section{México}

Correspondencia: Blanca Ivet Chávez Soto. 2a. Avenida, № 73, Col. El Sol, Cd. Nezahualcoyotl, Estado de México, México. E-mail: mil_chavez@hotmail.com

(C) Education \& Psychology $\mathrm{I}+\mathrm{D}+\mathrm{i}$ and Editorial EOS (Spain) 


\section{Resumen}

Introducción. El propósito de este estudio fue desarrollar e instrumentar un programa de enriquecimiento de la creatividad con actividades de juego educativo para alumnas sobresalientes de tercer grado de primaria de una zona marginada.

Método. Se utilizó un diseño pre test-post test. El pre test consistió en la identificación de los alumnos sobresalientes a través de la aplicación de tres instrumentos la Escala Farrens de Creatividad, la Escala de Compromiso con la Tarea y la Lista para Padres y Maestros para Identificar Potencial Sobresaliente, a través de los cuales se detectó a seis niñas con potencial sobresaliente. A estas alumnas se les aplicó la prueba WISC-R para complementar el perfil. Posteriormente, se desarrolló e instrumentó, por un periodo de 18 meses, un programa de enriquecimiento de la creatividad con 50 actividades de juego, constituido por 16 sesiones exploratorias, 16 de enriquecimiento I y 18 de enriquecimiento II. Al finalizar el programa, se realizó el post test con los instrumentos que se emplearon en la identificación, para determinar los cambios obtenidos por las alumnas.

Resultados. Los resultados de las sesiones exploratorias, de enriquecimiento I y II se analizaron con la prueba no paramétrica de Friedman y se encontraron cambios positivos en los indicadores de la creatividad verbal y gráfica. La evaluación pre y post test de los instrumentos se realizó con la prueba no paramétrica de Wilcoxon y un análisis de medidas repetidas con factor intrasujeto, la Prueba Farrens de Creatividad mostró que los dibujos de las alumnas después del programa fueron más elaborados y originales. En el Compromiso con la Tarea se encontró un decremento no significativo en el esfuerzo, interés y persistencia de las niñas en las actividades escolares. La prueba WISC-R mostró que el Cociente intelectual Total tuvo un incremento significativo, lo que indicó que las habilidades cognitivas aumentaron.

Discusión y Conclusión. Estos resultados resaltan la importancia de desarrollar programas de intervención que favorezcan las habilidades de los alumnos sobresalientes para promover un óptimo desarrollo educativo, emocional y psicológico.

Palabras Clave: sobresalientes, programa de enriquecimiento, juego, creatividad.

Recibido: 09/03/09 Aceptación inicial: 13/03/09 Aceptación final: 08/06/09 


\title{
Creativity enrichment program for gifted children from disadvantaged areas
}

\begin{abstract}
Introduction: The purpose of this study was to develop and apply a creativity enrichment program, with educative play activities, for third grade gifted children of elementary school in an economical margin zone.

Method: A pretest -postest designed was used. In pretest, three instruments were applied to identify gifted children: - Farren's scale Creativity (Zacatelco, 2005), Task Commitment scale (Zacatelco, 2005) and the List for Parents and Teachers to Identify Gifted Potential (Covarrubias, 2001), there were detected six girls with gifted potential, then WISC-R test was applied. A 18 months creativity enrichment program was applied whit 50 play activities: 16 explorer activities, 16 enrichment I, 18 enrichment II. To evaluate students' change a postest was applied.
\end{abstract}

Results: The results of the explorer sessions and enrichment I and II, were analyzed with no parametric test of Freedman, positive changes were found in verbal and graphic creativity. No parametric test of Wilcoxon and repeated measures test was applied to determine the changes before and after of the program. After the program, the Farren's scale of Creativity showed that the student's drawings were more original and elaborated, the Task Commitment scale showed no significant decrement in effort, interest and persistence in the activities, and the WISC-R test showed a significant increase in the total I.Q. This indicates that cognitive skills were increased.

Discussion or Conclusion: The results stand out the relevance of development programs to encourage the gifted student abilities to promote an excellent educative, emotional and psychological development.

Keywords: Gifted, enrichment program, play, creativity.

Received: 03/09/09 Initial Acceptance: 03/13/09 Final Acceptance: 06/08/09 


\section{Introducción}

A lo largo del tiempo han existido diferentes formas de conceptualizar a los alumnos con aprendizaje excepcional (Kirk y Gallagher, 1989), dentro de las cuales se han destacado términos como genios, precoces, superdotados y talentosos, estas diferencias se deben a que en algunas se ha considerado sólamente el cociente intelectual, en otras la creatividad, la motivación o el desempeño en diferentes áreas. De acuerdo, con Renzulli (1986), Ruiz (2003) y la Secretaría de Educación Pública (2006) el termino más utilizado es el sobresalientes, debido a que es más amplio y flexible, pues hace refencia aquellos alumnos que son persitentes, perseverantes, realizan contribuciones originales e innovadoras y además dominan algún campo específico (académico, científico-tecnológico, humanístico-social, artístico o deportivo).

En México la preocupación por atender a los alumnos sobresalientes se inició en el año de 1986, con el surgimiento del proyecto para niños con Capacidades y Aptitudes Sobresalientes (CAS), posteriormente, en 1989 y 1994 se estableció como uno de los objetivos la consolidación de dicho proyecto. Pero, lamentablemente el tema quedó olvidado a raíz de la reorientación de los servicios de educación especial en 1993 y, es hasta el año 2001 cuando nuevamente es retomado en el Programa Nacional de Educación en el apartado de política en expansión, el cual señaló como línea de acción el establecer los lineamientos para la atención de los sobresalientes y como meta para el 2002 el diseño de un modelo para brindar la educación necesaria para estos alumnos (Benavides, Maz, Castro y Blanco, 2004).

Calatayud, Forero y Ramírez (2007) coinciden en la necesidad de desarrollar una propuesta de intervención educativa para alumnos sobresalientes de escuelas públicas, de hecho, las autoras proponen un programa en el que consideran el diagnóstico y las estrategias de intervención educativa, dentro de las fases de su programa.

Actualmente, en el Distrito Federal se desarrolla el Programa de Niño Talento que consiste en proporcionar apoyo económico y algunos talleres a aquellos alumnos que han obtenido calificaciones de 9 y $10^{1}$ en sus notas escolares. Es importante mencionar que si bien se ha intentado proporcionar atención a los sobresalientes, el considerar sólo la calificación co-

\footnotetext{
${ }^{1}$ En México las calificaciones de los alumnos van de 0 a 10
} 
mo requisito para ingresar al programa resulta restrictivo, debido a que los alumnos sobresalientes muestran otras habilidades las cuales se deben tomar en cuenta para su identificación.

Lo anterior, reafirma el hecho de que en México aún falta mucho para poder brindarles la atención educativa que ellos necesitan. Esto concuerda con lo reportado por Benavides et al., (2004), Patton, Payne, Kauffman, Brown y Payne, (1991) y Valadez, Betancourt y Zavala (2005) quienes señalaron que a un grupo al que no se le han proporcionado las herramientas necesarias para alcanzar su máximo potencial es al de los sobresalientes, debido a que se considera que no requieren ajustes en la enseñanza por su facilidad para aprender. Es pertinente indicar que los sobresalientes precisan adaptaciones que optimicen su desarrollo y aprendizaje, que se conozcan sus características y sus necesidades educativas para que se diseñen programas que brinden apoyo para potencializar sus capacidades. Como señalaron Hernández y Borges (2005) el poseer altas capacidades intelectuales no es garantía de éxito, pues, en ocasiones las clases tradicionales y las tareas repetitivas provocan desajustes en el rendimiento académico de los sobresalientes.

Moska (2004) indicó que se deben identificar las necesidades educativas especiales que se encuentran asociadas a los niños sobresalientes, por lo que, se tienen que valorar sus capacidades e intereses. En este sentido, Zacatelco (2005) señaló que, cuanto antes se identifique y se intervenga con los niños sobresalientes en México mejor es el pronóstico, debido a que con la práctica y estimulación se desarrollan sus potencialidades. Robinson et al., (1981; citado en Acereda y Silva, 1998) demostraron en sus estudios, que los niños identificados tempranamente y que han participado en programas de intervención llegan a obtener grandes ganancias en su potencial sobresaliente.

De esta manera, la identificación es el primer paso para brindar la atención adecuada para estos alumnos, algunos autores como Acereda y Silva (1998); Alonso, Renzulli y Benito (2003); Benavides et al., (2004); Prieto (1999); Shea y Bauer (1999) concuerdan en que la definición propuesta por Renzulli (1986) en su Teoría de los Tres Anillos, constituye una base firme para seleccionar a los niños con potencialidad sobresaliente; desde esta perspectiva, se considera sobresaliente a aquella persona que presenta tres características personales:

- $\quad$ Alta creatividad -- Se emplea para describir a personas que realizan contribuciones sociales significativas y que generalmente es la originalidad, la novedad de la contribución lo que hace que se considere creativo (Zacatelco, 1994)--. 
- $\quad$ Elevado compromiso con la tarea -- Entendido como una refinada forma de motivación, es un proceso general energizante que dispara respuestas en el organismo para resolver un problema particular (tarea) en un área específica de desempeño (Castro, Oyanadel, Paez y Quintanilla, 2000) --.

- Habilidad intelectual superior -- Relacionada con la inteligencia, el éxito o el logro y con la aptitud académica. Renzulli (1986) consideró que los alumnos no necesariamente deben tener puntuaciones extremadamente altas en el cociente intelectual, al respecto Prieto (1999) mencionó que el modelo triádico establece que puede ser suficiente una desviación estándar, lo que equivale a una puntuación en el C.I. superior a 116 o un percentil superior a $75--$.

El objetivo de la identificación y evaluación de los alumnos sobresalientes, es establecer un punto de partida o línea base para conocer el desarrollo de cada uno en el área de su conocimiento y habilidades, para obtener una comprensión global y ofrecer una respuesta educativa más acorde a sus características. Por lo tanto, el evaluar conlleva a desarrollar alternativas de atención para favorecer las habilidades de los alumnos sobresalientes.

A lo largo del tiempo se han propuesto diversas estrategias de atención educativa para estos alumnos, tales como:

- La aceleración: Se basa en la idea de que el alumno puede aprender a un ritmo superior o más rápido en comparación con sus compañeros de la misma edad. Es una estrategia diseñada para que el alumno avance por el sistema educativo de forma flexible, lo cual implica saltarse algún grado escolar, permitir progresar a mayor velocidad que los demás y el terminar antes que sus compañeros la etapa escolar (Martín, 2004; Sánchez et al., 2006).

- El agrupamiento: Consiste en hacer grupos de alumnos sobresalientes de acuerdo a sus capacidades y proveerles de programas educativos adecuados a su nivel, esto se puede desarrollar dentro de las escuelas o en centro especiales (Castro et al., 2000). 
- $\quad$ El enriquecimiento: Algunos autores como Blanco (2001); Hernández (2004) concuerdan en que es una de las alternativas que más posibilidades ofrece a los alumnos sobresalientes. Se considera que ésta estrategia es sinónimo de la enseñanza individualizada debido a que se estructura de acuerdo a las características de los alumnos y mediante la cual se favorecen sus habilidades, además de que se puede aplicar de manera simultánea al currículum escolar (Martín, 2004; Sánchez et al., 2006 ).

Blanco (2001); Martín (2004); Sánchez et al., (2006); Valadez et al., (2005) señalaron que existen diferentes tipos de enriquecimiento, destacan el modelo de Triádico de Renzulli, el programa de enriquecimiento instrumental de Feuerstein, el modelo global de inteligencia de Pérez y Díaz, sólo por mencionar algunos. También es importante indicar que los modelos de enriquecimiento se pueden basar en las habilidades específicas que se encuentran asociadas a los alumnos sobresalientes como es el caso de la creatividad. En este sentido, Torrance (1977) señaló que la creatividad es un proceso que se expresa en los cambios que se descubren, es una capacidad susceptible a desarrollarse, y en los niños ésta se observa a través de sus producciones como lo son los cuentos, narraciones y dibujos. De acuerdo con Franco (2008) es importante favorecer esta capacidad en los niños para que puedan expresar sus ideas libremente, se favorezcan sus logros creativos y de esta manera tendrán una mejor calidad de vida.

Torrance (1977) estableció como indicadores de la creatividad: la fluidez -- capacidad para producir un gran número de ideas --, la flexibilidad -- aptitud para cambiar de una línea de pensamiento a otra--, la originalidad -- es la aptitud para aportar ideas o soluciones que están lejos de lo obvio, común o establecido -- y, la elaboración --aptitud para llenar de detalles las ideas--.

Para autores como Benito (1999); Betancourt y Valadez (2005) la creatividad es uno de los aspectos a considerar en los niños con potencial sobresaliente, ya que si ésta no se ve enriquecida se puede perder, además los autores enfatizaron que una de las formas para favorecer la creatividad es con el juego educativo, pues, se incrementa la intuición, la solución de problemas y la imaginación. Garaigordobil (1996) indicó que cuando el niño juega aprende y desarrolla su pensamiento debido a que ejercitan sus funciones cognitivas como la memoria, la comprensión, la concentración, la discriminación, la coordinación, sólo por mencionar algunas. Esto coincide con lo señalado por Vygotski (2006), quien mencionó que existen víncu- 
los entre el juego y el aprendizaje porque se crean áreas de desarrollo potencial a través de las cuales el niño tiene que emplear recursos diferentes para enfrentarse a las tareas y problemas.

Alonso y Aguirre (2004); Duarte (2003); Garaigordobil (2002); Prieto, López, Bermejo, Renzulli y Castejón (2002) han analizado los efectos del juego educativo en el desarrollo cognitivo y creativo de los niños, sus hallazgos muestran incrementos en la fluidez, flexibilidad, elaboración y originalidad de los alumnos, después de participar en los programas pues, manifiestan más ideas fuera de lo común y sus producciones están llenas de detalles.

Es importante indicar que el proveer las pautas educativas que necesitan los sobresalientes es indispensable y más aún cuando estos alumnos se encuentran en zonas urbanas marginales, debido a que en muchas ocasiones en estos lugares no se cuentan con los recursos económicos ni educativos para que ellos se desarrollen de manera integral. Autores como Gallagher (1994) han hecho hincapié en la importancia de identificar y atender a los sobresalientes que se encuentran en desventaja o en grupos de minorías, ya que en ocasiones éstos son ignorados y no tienen oportunidad de contar con otras alternativas educativas. En este sentido Zacatelco (2005) encontró que en México hacen falta trabajos que se orienten tanto a la detección e intervención de alumnos sobresalientes de zonas urbano marginales, consideró que la carencia de estos estudios se debe primordialmente a la falta de una definición adecuada y por ende a un sistema de identificación claro, a través del cual se detecte al sobresaliente, para diseñar programas o estrategias que permitan fomentar sus habilidades.

En este sentido, es importante resaltar que el enriquecimiento de la creatividad a través del juego educativo, favorece la capacidad sobresaliente, debido a que fomenta la curiosidad, el asumir riesgos, la imaginación y el desarrollo de otras habilidades cognitivas, con el empleo de diferentes recursos materiales y herramientas didácticas novedosas. De aquí que, el objetivo del presente trabajo consistió en desarrollar e instrumentar un programa de enriquecimiento de la creatividad con actividades de juego educativo para alumnas sobresalientes de tercer grado de primaria de una zona marginada. 


\section{Método}

\section{Participantes}

Seis alumnas identificadas con potencial sobresaliente, que presentaban alta creatividad, elevado compromiso con la tarea y con un cociente intelectual que iba de 118 - normal brillante -- a 125- superior -- una $M=121$, la edad promedio fue de 8.3 años, un rango de 8 9 años de edad, que cursaban el tercer grado de educación primaria. En cuanto a las familias de las niñas se encontró que, tres parejas estaban casadas, una vivía en unión libre, una de las madres era divorciada y la otra era madre soltera. Dos de las parejas mencionaron pertenecer a la religión cristiana y las otras cuatro eran católicas.

La edad promedio de las madres era de 34 años, con un rango de 28 a 42 años, en cuanto al nivel educativo se reportó que una concluyó la primaria, una tiene dos carreras técnicas -- enfermería y secretariado --, dos terminaron la preparatoria, una indicó que tenía carrera trunca de contaduría y otra termino la carrera de diseño gráfico. Tres de ellas se dedican al hogar, dos son comerciantes y una era vendedora ambulante. La edad de los padres de las niñas oscilaba entre de $\operatorname{los} 28$ y 50 años con una $M=$ de 36.6 años, se indicó que uno terminó la primaria, otro concluyó la secundaria, uno tiene carrera técnica -- enfermería --, otro cuenta con preparatoria y uno acabó la licenciatura -- medicina--. En cuanto a su ocupación se mencionó que uno es vendedor ambulante, otro es comerciante, uno es empleado, otro es enfermero y trabaja en salubridad, otro tiene un consultorio médico.

Se mencionó que cinco de las familias viven en casa dúplex y cuentan con todos los servicios -- agua, luz, teléfono y drenaje-- solamente una vive en un asentamiento irregular -un frente popular -- donde se carece de servicio telefónico.

\section{Instrumentos}

- Escala de Compromiso con la Tarea (Zacatelco, 2005), tiene como propósito identificar los niveles altos y bajos de este rasgo a partir de una dimensión general que es la motivación y los diferentes factores que la integran, tales como: el interés, la persistencia y el esfuerzo, así como su incidencia tanto en áreas curriculares --actividades en el salón de clase, tareas y exámenes -- como no curriculares - -deporte, lectura y música. La escala cuenta con 18 reactivos y tiene un nivel de confiabilidad $\alpha$ de .79 
- Prueba Farrens de Creatividad (Zacatelco, 2005), cuenta con 15 reactivos que exploran dos de los rasgos establecidos por Torrance (1972), que son Originalidad y Elaboración. Tiene una confiabilidad $\alpha$ de 90 . La actividad consiste en completar figuras a partir de un estímulo gráfico que le permita al alumno realizar ideas originales, novedosas y elaboradas.

- Lista para Padres y Maestros para Identificar Potencial Sobresaliente, diseñado por Covarrubias (2001), está dirigido a maestros para identificar el potencial sobresaliente en sus alumnos; dicho instrumento esta compuesto de 25 preguntas, las cuales se contestan dicotómicamente Sí o No.

- Escala de Inteligencia para Nivel Escolar Revisada (WISC- R Español): Tiene una confiabilidad 96 y mide la capacidad del niño para comprender y manejar su entorno, demostrando conducta inteligente en distintas formas. Consta de dos escalas -- verbal y ejecución -- y cada una está a su vez conformada por distintas subpruebas que evalúan diferentes capacidades como la agudeza visual, la memoria, la concentración, la comprensión, la capacidad de síntesis, el análisis, la organización, la destreza visomotora y el conocimiento de palabras. Las subpruebas de la escala verbal son: información, semejanzas, aritmética, vocabulario, comprensión y retención de dígitos, las subpruebas de la escala de ejecución son: figuras incompletas, ordenación de dibujos, diseño de cubos, composición de objetos, claves y laberintos (Wechsler, 1981)

- Programa de Enriquecimiento de la Creatividad: Constituido por un total de 50 actividades --16 exploratorias, 16 de enriquecimiento I y 18 de enriquecimiento II-- de juego educativo que tenían como propósito el favorecer la creatividad verbal y gráfica a través de la elaboración de cuentos, creación de historias, adivinanzas, acertijos, búsqueda de palabras y la realización de dibujos con diferentes materiales, que se desarrollaron en un tiempo aproximado de 18 meses -- dos actividades por semana con una duración de dos horas --.

Cada una de las sesiones exploratorias, de enriquecimiento I y II se calificaron con los criterios desarrollados por Torrance para evaluar la creatividad verbal y gráfica: 


\section{CREATIVIDAD VERBAL}

- FLUIDEZ: Se concede un punto por cada idea presentada, sin tomar en consideración las ideas repetidas y las no relevantes.

- FLEXIBILIDAD: Se valora el número de categorías o agrupamientos temáticos posibles de las ideas y se da un punto por cada categoría planteada.

- ORIGINALIDAD: Se da un punto en función de lo poco frecuente de la idea.

\section{CREATIVIDAD GRÁFICA}

- ORIGINALIDAD:

1 punto= Figura sencilla, trivial, esperada de acuerdo al entorno escolar que lo rodea.

2 puntos $=$ Figura con ideas diferentes a lo común.

3 puntos=Figura con elementos adicionales y con imaginación que se elabora a partir del estimulo.

- ELABORACIÓN:

1 punto $=$ Figura que se le añade un trazo o elemento.

2 puntos $=$ Figura con dos o cinco trazos o elementos.

3 puntos $=$ Figura con más de seis trazos o elementos.

\section{Contexto y escenario}

El estudio se realizó en una escuela primaria pública ubicada en la delegación Iztapalapa que se encuentra localizada al oriente del Distrito Federal. De acuerdo con los datos censales, es la delegación más poblada del país y tiene la mayor cantidad de adultos jóvenes. Además es el lugar donde residen más personas inmigrantes provenientes de distintos estados de la república (Instituto Nacional de Estadística y Geografía -INEGI-, 2006). En cuanto a la educación de la población se encontró que el 40\% de los habitantes es menor de 24 años y que se ha generado la infraestructura necesaria para atender la demanda educativa básica, lo que da como resultado un nivel bajo de analfabetismo. Sin embargo, es prudente señalar que el mayor porcentaje de analfabetismo en el Distrito Federal se ubica en esta demarcación.

El Instituto Ciudadano de Estudios sobre Inseguridad (2004) indicó que la delegación Iztapalapa es el segundo lugar con los niveles más altos en delitos en el Distrito Federal, además señalaron que las posibles causas que generan estos índices son la marginación y la concentración importante de unidades de manufactura, comercio y de servicio, lo que hace que diariamente haya un ingreso de más de 2 millones de personas que acude a los centros de trabajo, escuelas, comercios y espacios culturales.

Se realizó el estudio en la escuela Ejército de Oriente, que no cuenta con Unidad de Servicio de Apoyo a la Educación Regular (USAER) y por lo tanto, los niños con necesidades educativas especiales como los son los sobresalientes no son atendidos. 


\section{Procedimiento}

Se empleó un diseño pre test-post test: el pre test consistió en la identificación de los alumnos con potencial sobresaliente, posteriormente, a los alumnos detectados se les aplicó el programa de enriquecimiento de la creatividad y al finalizar después de 24 meses se realizó el post test en donde se aplicaron nuevamente los instrumentos empleados en la identificación, con el propósito de conocer los cambios de las alumnas.

En el pre test se aplicaron dos instrumentos a 99 alumnos de tercer grado: la Escala Farrens de Creatividad (Zacatelco, 1994) y la Escala de Compromiso con la Tarea (Zacatelco, 2005), participaron tres profesores que estaban a cargo de los grupos, los cuales respondieron la Lista para Padres y Maestros para Identificar Potencial Sobresaliente (Covarrubias, 2001), con los tres instrumentos aplicados se diseño una base de datos en el programa SPSS versión 10 y se obtuvo el percentil 75 a partir del cual se identificó a los alumnos que en dos de los tres instrumentos aplicados tuvieran una puntuación igual o superior a éste. Se detectó a seis alumnas que cumplían con este criterio, a las cuales se les aplicó la prueba WISC-R para complementar su perfil. Posteriormente se realizó una reunión en la escuela con los padres de las alumnas identificadas, se les expusieron los resultados de los instrumentos, se les explicó sobre las características y habilidades de sus hijas, así como de la importancia de favorecer sus capacidades a través de un programa educativo acorde sus necesidades educativas, al término de la reunión se solicitó su consentimiento informado para que sus hijas participaran en las actividades propuestas.

Concluida la fase del pre test se diseñó el programa en donde se consideró el perfil sobresaliente de las alumnas identificadas. Se encontró que las niñas tenían una elevada creatividad y para continuar favoreciéndola se desarrolló e instrumentó un programa de enriquecimiento de la creatividad a través de actividades de juego educativo, organizado en 50 sesiones de 2 horas cada una, 16 exploratorias -- con juegos diseñados para conocer la creatividad de las alumnas -- , 16 de enriquecimiento I -- actividades de juegos complejos que promovían la fluidez, la flexibilidad y la originalidad-- y 18 de enriquecimiento II -- juegos de acertijos verbales a través de los cuales se favorecía la creatividad verbal y gráfica-- que se desarrollaron a lo largo de 18 meses de forma grupal (Figura 1).

Al término del programa se realizó el post test en donde después de 24 meses se aplicaron nuevamente la Escala Farrens de Creatividad (Zacatelco, 2005), la Escala de Compro- 
miso con la Tarea (Zacatelco, 2005) y la prueba WISC-R, para identificar los cambios de las alumnas.

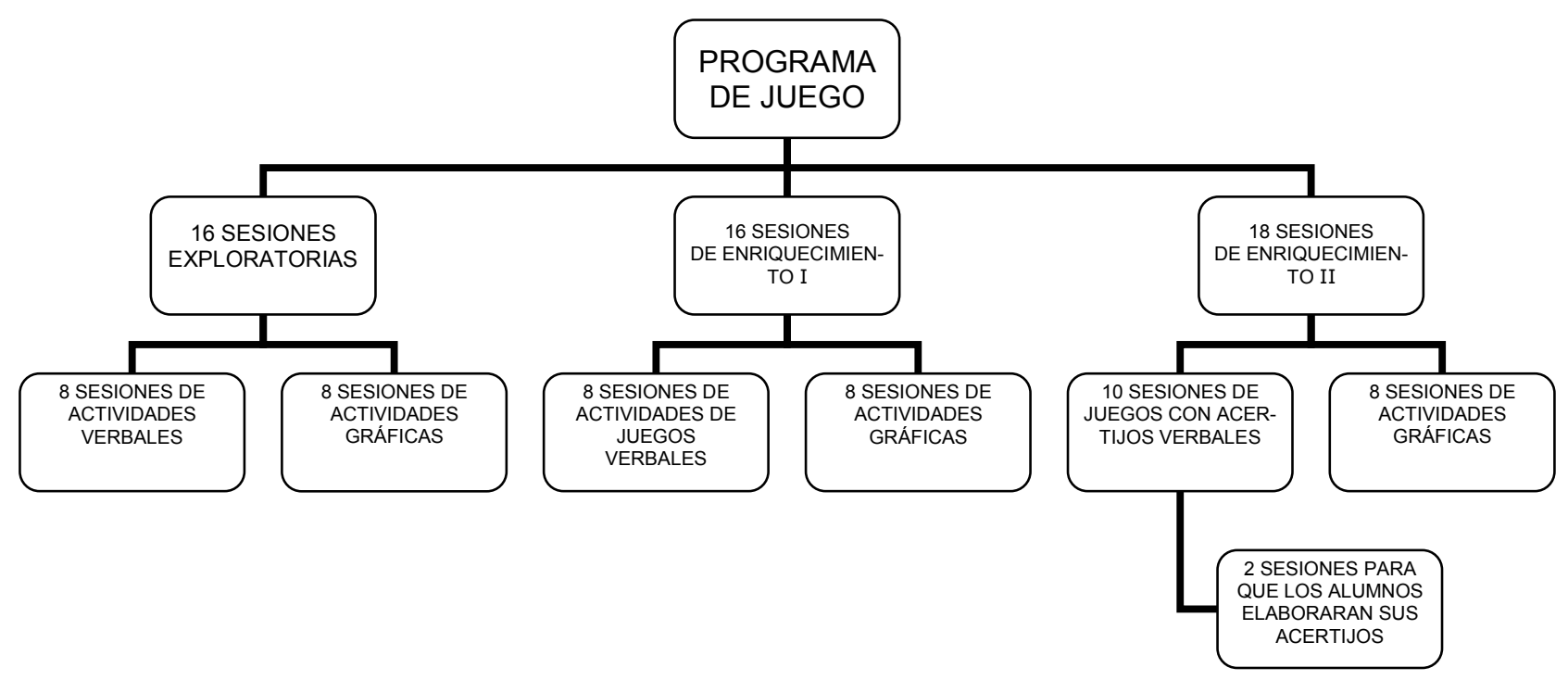

Figura 1. Distribución de las actividades del programa

\section{Análisis Estadístico}

Para determinar los cambios al finalizar el programa, se examinaron las medias de las sesiones exploratorias, de enriquecimiento I y II con la prueba no paramétrica de Friedman. También se analizaron las medias de las puntuaciones obtenidas en el pre test y post test de cada instrumento a través de la prueba no paramétrica de Wilcoxon, además se realizó un análisis de medidas repetidas con un factor intrasujeto.

\section{Resultados}

Resultados de las sesiones del programa

Con el propósio de identificar los cambios de las alumnas en las variables que componen la creatividad verbal y gráfica durante el desarrollo del programa, se compararon las medias de la fluidez, flexibilidad, originalidad y elaboración de las sesiones exploratorias, de enriquecimiento I y II a través de la prueba no paramétrica de Friedman. Se encontró que el programa de enriquecimiento favoreció la creatividad verbal de las alumnas al mostrar incrementos significativos en la puntuación general $(\mathrm{p}=.011$ con una $\alpha .05)$ y en las variables de fluidez ( $\mathrm{p}=.016$ con una $\alpha .05)$, flexibilidad $(\mathrm{p}=.002$ con una $\alpha .05)$ y originalidad $(\mathrm{p}=$ 
.002 con una $\alpha .05$ ), esto sugiere que las producciones escritas de las niñas en las sesiones de enriquecimiento I y II, presentaron mayor número de ideas que se ubican en diferentes categorias y son novedosas (Tabla 1).

Tabla 1. Medias y desviaciones éstandar de los componentes de la creatividad verbal durante las sesiones del programa.

\begin{tabular}{lcccccccc}
\hline \multicolumn{1}{c}{ Componentes } & \multicolumn{2}{c}{ Fluidez } & \multicolumn{2}{c}{ Flexibilidad } & \multicolumn{2}{c}{ Originalidad } & \multicolumn{2}{c}{$\begin{array}{c}\text { Creatividad } \\
\text { verbal }\end{array}$} \\
\cline { 2 - 8 } Sesiones & $M$ & $D . E$. & $M$ & $D . E$. & $M$ & $D . E$ & $M$ & $D . E$ \\
\hline Exploratorias & 152.83 & 39.08 & 44.67 & 6.25 & 31.67 & 10.42 & 272.5 & 51.88 \\
Enriquecimiento I & 184.33 & 23.83 & 99.83 & 10.72 & 43.5 & 8.78 & 284.33 & 26.33 \\
Enriquecimiento II & 262.67 & 38.65 & 208.83 & 35.79 & 93.67 & 15.13 & 565.17 & 86.77 \\
$\begin{array}{l}\text { Prueba de Fried- } \\
\text { man }\end{array}$ & $\begin{array}{c}\mathrm{p}=.016 \\
\alpha .05\end{array}$ & $\begin{array}{c}\mathrm{p}=.002 \\
\alpha .05\end{array}$ & $\begin{array}{c}\mathrm{p}=.002 \\
\alpha .05\end{array}$ & $\begin{array}{c}\mathrm{p}=.011 \\
\alpha .05\end{array}$ \\
\hline
\end{tabular}

Los efectos que se observaron a lo largo del programa en la creatividad gráfica se muestran en la Tabla 2, se encontraron cambios significativos en los componentes de elaboración $(\mathrm{p}=.004$ con una $\alpha .05)$ y originalidad $(\mathrm{p}=.002$ con una $\alpha .05)$, lo cual también se reflejo en la puntuación general de creatividad gráfica $(\mathrm{p}=.002$ con una $\alpha .05)$, esto indicó que los dibujos desarrollados por los alumnos en las sesiones de enriquecimiento, tenían muchos detalles y presentaban ideas interesantes.

Tabla 2. Medias y desviaciones éstandar de los componentes de la creatividad gráfica durante las sesiones del programa.

\begin{tabular}{lcccccc}
\hline \multirow{2}{*}{ Componentes } & \multicolumn{2}{c}{ Elaboración } & \multicolumn{2}{c}{ Originalidad } & \multicolumn{2}{c}{ Creatividad gráfica } \\
\cline { 2 - 7 } Sesiones & $M$ & $D . E$. & $M$ & $D . E$. & $M$ & $D . E$ \\
\hline Exploratorias & 23.67 & .52 & 21.33 & 1.37 & 45 & 1.55 \\
Enriquecimiento I & 24 & .02 & 24 & .02 & 48 & .02 \\
Enriquecimiento II & 30 & .01 & 30 & .01 & 60 & .01 \\
Prueba de Friedman & \multicolumn{2}{c}{$\mathrm{p}=.004$} & $\mathrm{p}=.002$ & \multicolumn{2}{c}{$\mathrm{p}=.002$} \\
& \multicolumn{2}{c}{$\alpha .05$} & \multicolumn{2}{c}{$\alpha .05$} & \multicolumn{2}{c}{$\alpha$} \\
\hline
\end{tabular}

Se observó que en las sesiones exploratorias, los dibujos elaborados por las alumnas tenían menos elementos y eran poco originales, mientras que los realizados en las sesiones de enriquecimiento I y II, mostraron la presencia de ideas poco usuales, gran riqueza y compleji- 
dad en los detalles que llenan de belleza los dibujos, además de presentar rasgos de movimiento, lo que los hace originales (Figura 2).

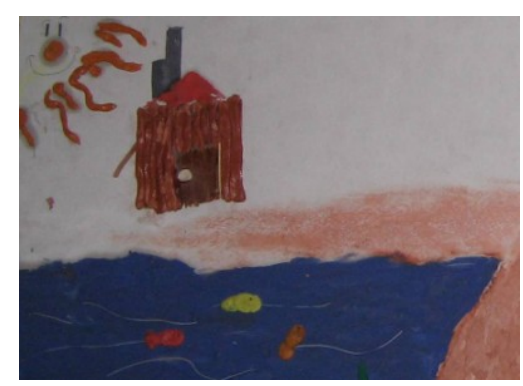

EXPLORATORIA

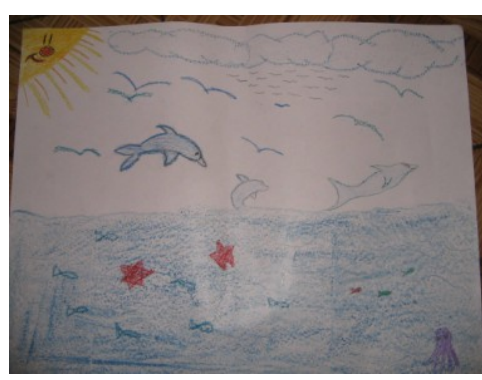

ENRIQUECIMIENTO I

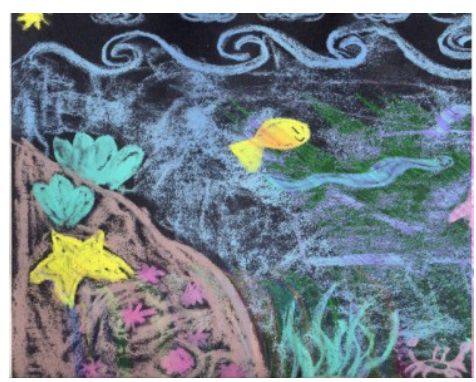

ENRIQUECIMIENTO II

Figura 2. Dibujos elaborados por los alumnos durante el programa.

\section{Resultados pretest-postets de los instrumentos}

En las medias de las puntaciones de pre test a post test de la prueba Farrens de Creatividad, se encontró un incremento significativo $\mathrm{p}=.028$ con una $\alpha .05$, lo cual también se observó en la originalidad $(\mathrm{p}=.027$ con una $\alpha .05)$, en cuanto a la variable de elaboración no presentó cambios significativos $(\mathrm{p}=.180$ con una $\alpha .05)$ estos resultados indicaron que los dibujos desarrollados por las alumnas después del programa de enriquecimiento fueron más originales y elaborados (Tabla 3). Se realizó un análisis de medidas repetidas para determinar los cambios intrasujeto y se encontró un incremento significativo $F_{(1,5)}=.009, \mathrm{p}<.05$, lo que indicó que la creatividad gráfica al finalizar el programa aumentó en las seis alumnas.

Tabla 3. Medias y desviaciones éstandar de los componentes de la creatividad gráfica del pre test y post test de la Prueba Farrens.

\begin{tabular}{lcccccc}
\hline \multirow{2}{*}{ Componentes } & \multicolumn{2}{c}{ Elaboración } & \multicolumn{2}{c}{ Originalidad } & \multicolumn{2}{c}{ Creatividad gráfica } \\
\cline { 2 - 6 } Prueba Farrens & $M$ & $D . E$. & $M$ & $D . E$. & $M$ & $D . E$ \\
\hline Pretest & 43 & 2.61 & 25.83 & 11.65 & 48.50 & 24.92 \\
Postest & 44 & 2.32 & 38.83 & 6.11 & 81 & 8.17 \\
Prueba de Wilcoxon & \multicolumn{2}{c}{$\mathrm{P}=.180$} & $\mathrm{p}=.027$ & $\mathrm{P}=.028$ \\
& \multicolumn{2}{c}{$\alpha .05$} & \multicolumn{2}{c}{$\alpha .05$} & \multicolumn{2}{c}{$\alpha$} \\
\hline
\end{tabular}

Al comparar las medias de la variable Compromiso con la Tarea se observó una disminución en las puntuaciones obtenidas del pre test $(M=88.3)$ al post test $(M=85.3)$, la prueba de Wilcoxon reportó que estas diferencias no eran significativas $\mathrm{p}=.345$ con una $\alpha$ .05 , lo cual mostró un decremento en el interés, esfuerzo y motivación de los alumnos hacia 
las actividades escolares. En cuanto al análisis de medidas repetidas para determinar los cambios intrasujeto se encontró que no hubo cambios significativos $\mathrm{F}_{(1,5)}=.722$, $\mathrm{p}<.05$, al finalizar el programa en cada una de las alumnas.

Para determinar los efectos del programa en la variable inteligencia, se realizó la prueba no paramétrica de Wilcoxon con las puntuaciones pres test - post test de la prueba WISC$\mathrm{R}$, se encontró que el C. I. total tuvo un incremento significativo $(p=.028$ con una $\alpha .05)$ de pre test $(M=121)$ a post test $(M=141.6)$, lo que indicó que las habilidades cognitivas de las alumnas incrementaron después del programa. Posteriormente, se analizaron las subescalas de la prueba WISC-R y se observó que el C. I. verbal incrementó significativamente con una p = .046 y una $\alpha .05$, y el C. I. de ejecución aumentó con una diferencia significativa de $p=.027$ con una $\alpha .05$ (Figura 3). Estos resultados sugieren que el programa favoreció habilidades como la memoria, la percepción, discriminación visual, conocimiento de palabras y desarrollo de lenguaje de las alumnas.

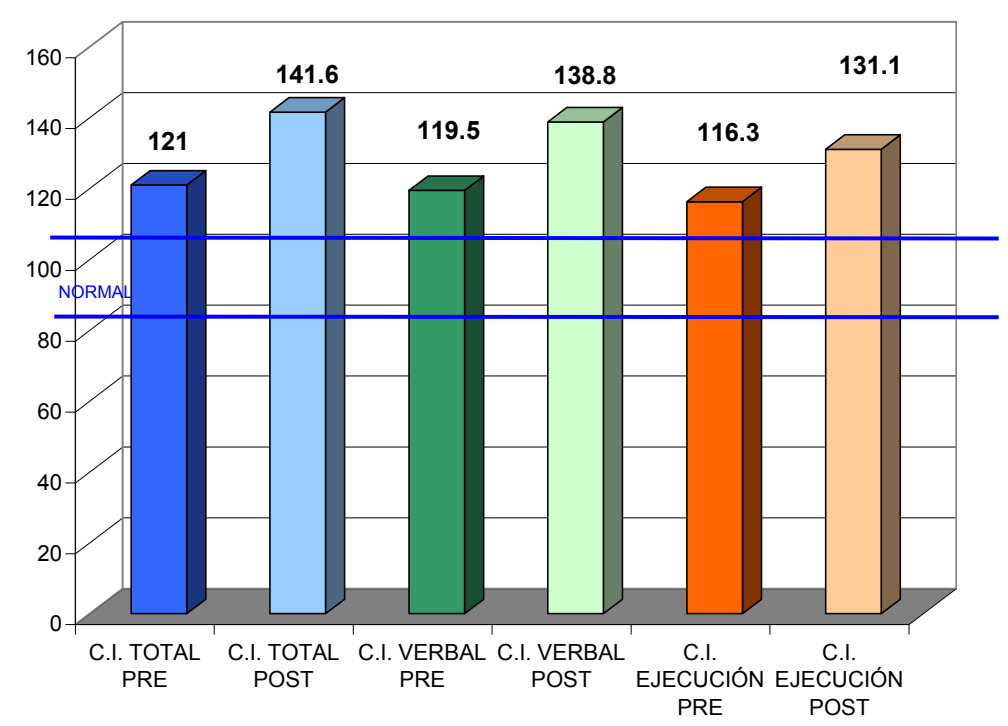

Figura 3. Medias de los C.I. pre test y post test de la prueba WISC-R.

Se utilizó la prueba no parametrica de Wilcoxon para conocer los cambios de la escala verbal y de ejecución. En la escala verbal se encontró un incremento significativo en las subescalas de información $(\mathrm{p}=.026$ con una $\alpha .05)$, semejanzas $(\mathrm{p}=.046$ con una $\alpha .05)$, comprensión $(\mathrm{p}=.042$ con una $\alpha .05)$ y retención de dígitos $(\mathrm{p}=.041$ con una $\alpha .05)$, lo que indicó que aumentó la comprensión, el conocimiento y entendimiento de palabras, de tal forma que, se favorecieron las habilidades verbales del pensamiento. En escala de ejecución se observó un incremento significativo en las medias de pretest y posttest en las sub escalas de fi- 
guras incompletas $(\mathrm{p}=.026$ con una $\alpha .05)$, composición de objetos $(\mathrm{p}=.042$ con una $\alpha .05)$ y laberintos $(\mathrm{p}=.046$ con una $\alpha .05)$, esto sugirió que las alumnas aumentaron su discriminación visual, coordinación visomotora, organización visual y de concentración (Tabla 4).

Tabla 4. C.I. total, verbal, de ejecución y de las alumnas antes y después del programa.

\begin{tabular}{|c|c|c|c|c|c|c|}
\hline \multirow{2}{*}{ ESCALAS } & \multirow[t]{2}{*}{ SUB ESCALAS } & \multicolumn{2}{|c|}{ PRE TEST } & \multicolumn{2}{|c|}{ POST TEST } & \multirow[t]{2}{*}{ WILCOXON } \\
\hline & & $M$ & D.E & $M$ & D.E & \\
\hline \multirow{6}{*}{ VERBAL } & Información & 10.83 & 1.72 & 15.17 & 1.33 & $\begin{array}{c}p=.026 \\
\alpha .05\end{array}$ \\
\hline & Semejanzas & 12.33 & 2.42 & 18.00 & 2.45 & $\begin{array}{c}p=.046 \\
\alpha .05\end{array}$ \\
\hline & Aritmética & 13.50 & .84 & 14.33 & 2.16 & $\begin{array}{c}\mathrm{p}=.414 \\
\alpha .05\end{array}$ \\
\hline & Vocabulario & 15.50 & 2.66 & 17.67 & 2.16 & $\begin{array}{c}p=.078 \\
\alpha .05\end{array}$ \\
\hline & Comprensión & 14.17 & 2.23 & 16.00 & 1.55 & $\begin{array}{c}\mathrm{p}=.042 \\
\alpha .05\end{array}$ \\
\hline & Retención de dígitos & 9.00 & 2.28 & 12.33 & 3.88 & $\begin{array}{c}p=.041 \\
\alpha .05\end{array}$ \\
\hline \multirow{6}{*}{ EJECUCIÓN } & Figuras incompletas & 11.17 & 2.04 & 13.83 & 2.99 & $\begin{array}{c}\mathrm{p}=.026 \\
\alpha .05\end{array}$ \\
\hline & Ordenación de dibujos & 13.67 & 1.97 & 14.00 & 1.79 & $\begin{array}{c}\mathrm{p}=.590 \\
\alpha .05\end{array}$ \\
\hline & Diseño de cubos & 12.50 & 2.07 & 13.83 & 2.79 & $\begin{array}{c}p=.167 \\
\alpha .05\end{array}$ \\
\hline & Composición de objetos & 10.83 & 2.40 & 14.50 & 3.83 & $\begin{array}{c}\mathrm{p}=.042 \\
\alpha .05\end{array}$ \\
\hline & Claves & 13.17 & 1.60 & 15.50 & 3.27 & $\begin{array}{c}p=.072 \\
\alpha .05\end{array}$ \\
\hline & Laberintos & 12.17 & 1.94 & 18.00 & 2.45 & $\begin{array}{c}p=.046 \\
\alpha .05\end{array}$ \\
\hline
\end{tabular}

En la tabla 5 se observan los efectos que tuvo el programa sobre el C.I. total, verbal y de ejecución de cada una de las alumnas. Se encontró que el C.I. total y verbal mostraron un incremento, cinco de las seis niñas tienen actualmente un cociente intelectual superior a 130 lo que equivale a un rango de "Muy Superior", sólo una alumna obtuvo una puntuación menor a 120, lo cual la coloca en un rango de "Superior". En cuanto al C.I.de Ejecución se observó que tres alumnas obtuvieron una puntuación mayor a 130 (Muy superior) y las otras tres mostraron una puntuación mayor a 121 (Superior), estos datos indican que aumentaron las habilidades cognitivas de las alumnas después del programa. Además se realizó el análisis de medidas repetidas para determinar los cambios intrasujeto y se encontró un incremento significati- 
vo $\mathrm{F}_{(1,5)}=.004 \mathrm{p}<.05$, lo que indicó que el cociente intelectual de las seis alumnas aumento al finalizar el programa.

Tabla 5. C.I. total, verbal, de ejecución y de las alumnas antes y después del programa.

\begin{tabular}{ccccccc}
\hline \multirow{2}{*}{ INICIALES } & \multicolumn{2}{c}{ C.I. TOTAL } & \multicolumn{2}{c}{ C.I. VERBAL } & \multicolumn{2}{c}{ C.I. EJECUCIÓN } \\
\cline { 2 - 6 } & ANTES & DESPUÉS & ANTES & DESPUÉS & ANTES & DESPUÉS \\
\hline B.R & 119 & 142 & 115 & 147 & 117 & 129 \\
A.E & 125 & 137 & 133 & 143 & 111 & 121 \\
L.Y. & 121 & 142 & 127 & 142 & 109 & 130 \\
B.C. & 118 & 121 & 120 & 119 & 111 & 121 \\
A.P. & 118 & 140 & 107 & 136 & 120 & 135 \\
S.V. & 125 & 153 & 115 & 146 & 130 & 151 \\
\hline
\end{tabular}

\section{Discusión y Conclusiones}

Autores como Guilford (1981) y Torrance (1977) señalaron que la creatividad ha sido considerada como un proceso que integra elementos necesarios para desarrollar habilidades intelectuales, por lo que, le dan gran importancia al papel del pensamiento en la actividad creadora, donde se trata de explicar "ese cambio cualitativo" que posibilita la producción de lo nuevo. A este respecto Vygotski (1999) resalta la idea de que, la creatividad se expresa en cualquier tipo de actividad del hombre cuando crea algo nuevo. Este mismo autor planteó que en el caso específico de los niños, la creatividad se manifiesta a través del juego y que es el tipo preferido de creación a edades tempranas. En este sentido, Torrance (1977) manifestó que a medida que el niño crece se disminuye la creatividad y hasta se corre el riesgo de que desaparezca si no es estimulada a tiempo.

De ahí que el presente estudio se centró en el diseñó e instrumentación de un programa de enriquecimiento de la creatividad a través del juego educativo, pues, es importante favorecer esta capacidad en edad escolar para que los niños puedan expresar sus ideas libremente y desarrollen habilidades que ayudarán a la adquisición de otras capacidades.

Cabe señalar que el primer paso para brindar la atención educativa a los sobresalientes es la identificación temprana, diversos autores como Sánchez (2003); Moska (2004); Acereda y Silva (1998); Benito (2004), coinciden en que cuanto antes se identifique a los alumnos sobresalientes mejor es el pronóstico para evitar que sus habilidades se pierdan. De esta manera 
se consideró importante detectar a los alumnos con potencial sobresaliente a través del Modelo para la identificación desarrollado por Zacatelco (2005) en el que se evalúan aspectos como la creatividad, el compromiso con la tarea y la nominación del maestro, dicho modelo fue validado en escuelas de educación primara de la delegación Iztapalapa, ubicadas en una zona de alta marginación.

Este modelo se retomó para realizar en un primer momento la identificación de niños sobresalientes de una zona marginal y se encontró que un $6 \%$ de estos presentaban un claro perfil sobresaliente -- elevada creatividad, compromiso con la tarea, cociente intelectual que iba de 118 (normal brillante) a 125 (superior) --, estos datos coinciden con lo reportado por autores como Davis y Rimm (1989); Jiménez, Artiles, Ramírez, y Alvarez, (2004); Renzulli, (1986); Secretaria de Educación Pública (2006); Valadez (2004) y Zacatelco (2005) quienes reportan una incidencia de alumnos sobresalientes en escuelas primarias que oscila de un 3\% a un $24 \%$. Con lo anterior, se reafirma el hecho de que dentro de las escuelas públicas existen alumnos con potencial sobresaliente, lo que lleva a reflexionar sobre la importancia de la identificación oportuna de éstos niños, en especial si habitan en zonas marginadas en las que sus padres carecen de elementos para estimular dicho potencial. Se debe destacar que el desarrollo psicológico y educativo del sobresaliente se da a través de la interacción de distintos factores socioculturales (familia, escuela, sociedad) y psicosociales, que de acuerdo con Mönks (1999) son determinantes en el aprendizaje del niño.

Cabe señalar que el programa de enriquecimiento que posteriormente se desarrolló de manera grupal, favoreció el aprendizaje cooperativo, el trabajo en equipo y que las niñas intervinieran de manera autónoma en las actividades (Alonso et al., 2003). En este sentido, Rogoff (1993) destaca la importancia del aprendizaje a través de la participación en un grupo social porque se generan situaciones de interrelación, se favorece la comunicación y se enfrenta al niño a situaciones que implican un problema o reto lo cual hace que desarrollen destrezas indispensables en las habilidades cognitivas.

Los efectos del programa en las niñas participantes mostraron un incremento en la manifestación de la creatividad verbal y gráfica después de concluir las actividades de enriquecimiento, pues, sus producciones verbales y sus dibujos fueron más elaborados, presentaban muchos detalles que embellecían sus obras, con ideas poco usuales, con gran riqueza y complejidad, además de que algunos dibujos tenían rasgos de movimiento, aspectos que no se 
observaron en los diseños de las sesiones exploratorias, es decir, mostraron creaciones que se alejaban de lo obvio o lo común. Estos datos confirman lo planteado por algunos teóricos de la creatividad como Alonso y Aguirre (2004); Duarte (2003); Garaigordobil (2002); Prieto, et al., (2002) quienes reportaron resultados similares, es decir, efectos positivos en los indicadores de la creatividad después de instrumentar programas para enriquecerla a través de actividades lúdicas.

Respecto al compromiso con la tarea destaca el hecho de que las niñas al finalizar la intervención manifestaron que las actividades escolares les provocaban poco interés y motivación, por lo que su esfuerzo y persistencia por realizarlas disminuyó; lo cual se puede deber a que en ocasiones las características del proceso educativo, las actividades escolares y las clases tradicionales son aburridas y hacen que se cause desmotivación general (Hernández y Borges, 2005; Pomar, 1998). De esta manera, se debe considerar lo señalado por Fernández Ongoratoy Casullo (2004); Kondor (2007); Li (1998); Suárez y Fernández (2005) quienes enfatizaron la importancia de mantener una alta motivación debido a que ésta se relaciona con el proceso de aprendizaje y que se establece a partir de las propias necesidades e intereses de los alumnos para que logren disfrutar su aprendizaje a través de un compromiso serio con proyectos escolares y de esta manera fomentar una mayor motivación hacia tareas o actividades de la escuela.

Por otro lado, se dio un incremento significativo en la inteligencia total, registrada a través de la prueba WISC-R. En las escalas verbal y de ejecución, se observó que las subescalas que más contribuyeron fueron las de información, semejanzas, comprensión, retención de dígitos, figuras incompletas, composición de objetos y laberintos; lo cual indicó que los alumnos aumentaron su comprensión verbal, el conocimiento, entendimiento de palabras, la discriminación visual, coordinación visomotora, organización visual y concentración.

Autores como Galton (1883, citado en Gardner, 2001) y Terman (1921, citado en Prieto, 1999) señalaron que la inteligencia es innata, que se hereda de padres a hijos y que una persona poco puede hacer para alterar el potencial intelectual con el que se ha nacido. En la actualidad esta concepción ha cambiado, al considerar que si bien los alumnos sobresalientes nacen con una inteligencia elevada, se requiere de un ambiente adecuado y de programas acordes con las necesidades específicas de ésta población para que no se pierdan sus capacidades (Cavazos, 2007). En este sentido, los resultados obtenidos en ésta investigación en los 
que se observa un incremento significativo en las habilidades cognitivas de las seis alumnas, lleva a reconocer la influencia de factores externos como el ambiente, la familia, la educación y los programas de apoyo que pueden tener estos alumnos para favorecer sus capacidades. Lo que confirma lo señalado por Betancourt (2004) quién indicó que los programas para favorecer las habilidades cognitivas de los alumnos sobresalientes deben basarse en una educación creativa e inteligente que les propicie la oportunidad de desarrollar sus capacidades, fortalecer su pensamiento y fomentar sus valores.

De la misma manera, cabe destacar la participación que de manera indirecta tuvieron los padres pues éstos se mostraron interesados y muy comprometidos con esta propuesta de trabajo al saber que sus hijas tenían potencial sobresaliente les proveyeron de recursos adicionales para favorecer sus habilidades, a pesar de sus limitaciones económicas, por ejemplo; una alumna tomaba clases de natación, otra se inscribió a inglés y dos de las niñas asistían a ballet. Así, el hecho de saber que ellas tenían estas capacidades, los motivó a buscar alternativas adicionales para su educación, por lo que no se puede negar que la combinación de todos estos factores se reflejó en los cambios observados en las menores al finalizar el programa de enriquecimiento de la creatividad.

Esto también lleva a considerar la importancia de desarrollar programas dirigidos a los padres de los sobresalientes que habitan en zonas marginadas, a través de los cuales se les proporcione información sobre las capacidades, los talentos, las propuestas educativas y las actividades que ellos pueden realizar para favorecer las habilidades de sus hijos de acuerdo a sus propias condiciones. Autores como Martín (2004) han señalado que los padres pueden reaccionar de diferentes formas al conocer que sus hijos son sobresalientes, sus conductas pueden ir desde expresar preocupación, ansiedad, confusión o incluso sentirse frustrados al no saber que hacer o en el caso contrario reaccionan de tal forma que les programan actividades adicionales al salir del colegio y exigirles buenas calificaciones. En este sentido, es indispensable el proponer que en estudios posteriores se considere el desarrollo de programas enfocados a los padres de familia de zonas marginada para que brinden a sus hijos experiencias educativas afines a sus capacidades y condiciones socioeconómicas.

De esta manera, los hallazgos, ponen de manifiesto la importancia de realizar una identificación temprana de los menores sobresalientes que se encuentran en escuelas públicas de zonas marginadas, a través de un modelo que considere sus características, los factores 
ambientales y culturales que los rodean. Como se aprecia en el presente estudio existen alumnos sobresalientes en estas zonas, a los que en general no se les proporcionan los recursos necesarios para una educación de calidad, debido tanto a que no se conocen sus características y habilidades así como a que habitan en ambientes poco estimulantes y les pone en situación de riesgo al no ser detectados, por lo que su potencial puede disminuir o se canalizarse en actividades antisociales. De ahí la relevancia de brindar alternativas de aprendizaje acordes a las necesidades educativas de estos alumnos para que se desarrollen de una manera integral y logren un mejor bienestar académico, psicológico, emocional y social. 


\section{Referencias}

Acereda, A. y Silva S. (1998). La superdotación. España: Síntesis Psicológicas

Alonso, L. y Aguirre, R. R. (2004). La escritura creativa en la escuela: Una experiencia pedagógica (de; con) juegos lingüísticos y metáforas. Revista de Pedagogía, 74 (XXV), 375-399.

Alonso, J., Renzulli, J. y Benito, Y. (2003). Manual Internacional de Superdotados. Manual para Profesores y Padres. España: Editorial EOS.

Benavides, M., Maz, A., Castro, E. y Blanco, R. (2004). La Educación de Niños con Talento en Iberoamérica. Oficina Regional de Educación para América latina y el Caribe. Chile: Trineo.

Benito, M. Y. (1999). ¿Existen los superdotados?. España: Praxis.

Benito, M. Y. (2004) Ventajas de la detección temprana del niño con talento y superdotado [Versión electrónica], Educar, 29, 9-16.

Betancourt, M. J. (2004). Una dieta balanceada para los alumnos sobresalientes y de altas capacidades [Versión electrónica], Educar,29, 9-16.

Betancourt, M. J. y Valadez S. M. D. (2005). Atmósferas Creativas. Juega, piensa y crea. México: Manual moderno.

Blanco, V. M. C. (2001). Guía para la identificación y seguimiento de alumnos superdotados. España: CISS Praxis Educación.

Calatayud, M. A., Forero, L.A. y Ramírez, G. M. C. (2007). Proyecto de investigación e innovación: Una propuesta de intervención educativa para alumnos y alumnas con aptitudes sobresalientes [Versión electrónica], Faísca, 12(14), 57-83.

Castro, P., Oyanadel, C., Páez, A. y Quintanilla, R. (2000). Implicaciones de una educación especial para superdotados. Recuperado el día 25 de mayo de 2006, desde: http:/www. mixcuatros.com/prgtalent.htlm

Cavazos. A. (2007). Niños con potencial sobresaliente. Recuperado el día 15 de abril de 2008, desde: http:/elporvenir.com.mx/ notas.asp?.nota_id=146964

Covarruvias, P. (2001). Características cognitivas y socioafectivas de los niños y niñas sobresalientes de la zona norte de México. Tesis de Maestría no publicada, Universidad Nacional Autónoma de Tlaxcala. Departamento de Educación Especializada. Tlaxcala. México.

Davis, G. y Rimm, S. (1989) Education of the gifted and talented. New Jersey: Prentice Hall 
Duarte, B. E. (2003). Creatividad como un recurso psicológico para niños con necesidades educativas especiales. Recuperado el día 12 de mayo de 2007, desde: dialnet. unirioja.es/servlet/oaiart? Código $=1290555$

Fernández, M., Ongorato, P. y Casullo, M. (2004) Adaptación y validación de una escala sobre clima motivacional de clase. Psicología y Ciencia Social, 6(22),13-22.

Franco, J. C. (2008). Creative relaxation, motor creativity, self-concept in a sample of children from Early Childhood Education. Electronic Journal of Research in Educational Psychology, 14, 6(1), 29-50.

Gallagher, J. (1994). Teaching the gifted child. Boston: AllynyBacon. Inc.

Gardner, H. (2001). La inteligencia reformulada. Las inteligencias múltiples en el siglo XXI. España: Paidós.

Garaigordobil, L. M. (1996). Psicología para el desarrollo de la cooperación. España: Esclée De Brouwer.

Garaigordobil, L. M. (2002). Efectos de la Participación en el programa de arte Ikertze sobre la creatividad verbal y Gráfica. Anales de psicología, 18 (julio), 95-110.

Guilford, J. (1981) Tres aspectos del Intelecto. New York: Irvington Publisher.

Hernández, A. G. (2004). Intervención educativa para niños con necesidades educativas especiales asociadas a las altas capacidades [Versión electrónica], Educar, 29, 67-74.

Hernández, J. C. y Borges, R. A. (2005). A self-regulated learning program for high-ability individuals, using on-line tools. Electronic Journal of Research in Educational Psychology, 7, 3(3), 233-252.

INEGI (2006). Estados Unidos Mexicanos, resultados preliminares, XII censo de población y vivienda.

Instituto Ciudadano de Estudios sobre Inseguridad (2004). Recuperado el día 20 de agosto de 2008, desde: www.icesi.org.mx

Jiménez, J. E., Artiles, C. Ramírez, G. y Alvarez, J. (2004) Modelo de identificación temprana del alumnado con alta capacidad intelectual en la comunidad autónoma de canarias. Infancia y aprendizaje, 27 (4), 469-483.

Kirk, S. A. y Gallagher, J. J. (1989) Educating Exceptional. U.S.A: Houghton.

Kondor, C. (2007) The Effects on Differentied Instruction on the Motivation of talented and Gifted Students. Recuperado el 14 de agosto de 2008 de: http://www.eric. ed.gov/ERICDocs/data/ericdocs2sq1/content_torage_01/0000019b/80/2b/c3/69.pdf

Li, A. (1998). Self-perception and motivational orientation in gifted children. Roeper Review, $10(3), 175-180$. 
Martín, L. M. P. (2004). Niños inteligentes. España: Palabra.

Mönks J. F. (1999) Respuestas Educativas para Alumnos Superdotados y Talentosos. Zaragoza: Mira Editores.

Moska, E. L. (2004). Identificación de los niños CAS [versión electrónica]. Educar, 29, 1734.

Patton, R. J., Payne, S. J., Kauffman M. J., Brown, B. G. y Payne A. R. (1991). Casos de Educación especial. (pp. 189-209). México: Limusa.

Pomar, C. (1998). Desmotivación Académica. Recuperado el día 30 de Abril de 2007, desde: http://www.congreso/superdotado integral./htm

Prieto, D. (1999). Identificación, Evaluación y Atención a la Diversidad del Superdotado. España: Aljibe.

Prieto, D., López, O., Bermejo, M., Renzulli, J. y Castejón, J. (2002). Evaluación de un programa de desarrollo de la creatividad. Psicothema, 14(2), 410-414.

Renzulli, S. J. (1986). Systems and Models for Developing Programs for the Gifted and Talented. USA: Creative Learning Press.

Rogoff, B. (1993). Aprendices del pensamiento. España: Paidós.

Ruiz, L. (2003) Notas para una definición del individuo sobresaliente. Recuperado el día 23 de mayo de 2007 de: http://proyecto-cas.iespana.es/document/Ira2.htm

Sánchez, M. E. (2003) Los niños superdotados: Una aproximación a su realidad. Recuperado el día 11 de mayo de 2007 de: www.dmenor-ad.es/pdf/publicaciones/los _niños_superdotado.pdf

Sánchez, P., Medrano, C., Martín, S., Puc, D., Váldes, F., Jiménez, S., Torres, B., García, G., Cárdenas, C., Ac Cauich, A., Viana, V., Cima C. y Leal. K. (2006). Detección y registro de niños de secundaria con capacidad sobresaliente en zonas rurales y suburbanos del estado de Yucatán. México: CONCYTEY, UADY y CONACYT.

Secretaria de Educación Pública, Dirección de Innovación Educativa (2006) Orientaciones Generales para el Funcionamiento de los Servicios de Educación Especial. México: Secretaria de Educación Pública.

Shea, M. T. y Bauer, M. A. (1999). Educación Especial un Enfoque Ecológico. México: Mc Graw-Hill.

Suárez, J. y Fernández, A. (2005). Escalas de evaluación de las estrategias motivacionales de los estudiantes. Anales de psicología, 21(1), 116-128.

Torrance, P. (1977). Educación y capacidad creativa. USA: Prentice Hall 
Valadez, S. M. D. (2004) Niñas, superdotación y contexto social [Versión electrónica], Educar, 29, 53-58.

Valadez, S. M. D., Betancourt, M. J. y Zavala B. M. A. (2005). Alumnos Superdotados y Talentosos. Identificación, Evaluación e Intervención Una Perspectiva para Docentes. México: Manual Moderno.

Wechsler, D. (1981) Escala de inteligencia para el nivel escolar - revisada (wisc-r español)

Vygotski, S. L.(1999). Imaginación y creación en la edad infantil. Cuba: Pueblo y Educación.

Vygotski, S. L. (2006). El desarrollo de los procesos psicológicos superiores. España: Crítica

Zacatelco, R. F. J. (1994) El potencial creativo en un grupo de niños de primer grado, de clase popular baja del D.F., Tesis Maestría en Psicología (Educación Especial) no publicada, Facultad de Estudios Superiores Zaragoza, UNAM, México.

Zacaltelco, R. F. J. (2005). Modelo para la Identificación del niño sobresaliente en escuelas de educación primaria, Tesis de Doctorado en Psicología (Educación Especial), No publicada Facultad de Estudios Superiores Zaragoza, Universidad Nacional Autónoma de México. Distrito Federal. México. 
Anexos. Ejemplos de actividades.

\section{Ejemplo de actividad exploratoria \\ (Creatividad Verbal)}

\section{NUEVOS TÍTULOS PARA VIEJOS CUENTOS}

\section{OBJETIVO:}

望 Estimular la fluidez, flexibilidad y la originalidad en el área verbal, a través de inventar títulos nuevos para cuentos que ya se conocen.

\section{PROCEDIMIENTO:}

Se organizan por parejas.

望 Se colocan dentro de la bolsa las papeletas con los nombres de los cuentos (Blanca nieves y los siete enanos, Caperucita roja, Hansel y Gretel, Los tres cerditos, Cenicienta, El gato con botas).

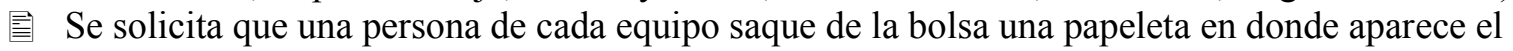
cuento que les ha tocado, y que lo comenten solamente con su pareja.

咅 Se les indica que el juego consiste en crear nuevos títulos para el cuento que les toco.

咅 Se les sugiere que cada equipo realice sus títulos tratando que sean divertidos y originales.

害 Posteriormente se reúnen todos los integrantes formando un círculo y se dicen los nuevos títulos que han pensado.

兽 Se indica que traten de adivinar el nombre del cuento a partir de los títulos nuevos que han creado sus compañeros.

\section{Ejemplo de actividad de enriquecimientoI \\ (Creatividad Gráfica) \\ ¿QUÉ ES Y DÓNDE ESTÁ?}

\section{OBJETIVO:}

* Desarrollar la creatividad gráfica a través de producir ideas y plasmarlas en un dibujo tomando como base un estímulo (hoja con una figura geométrica)*.

\section{PROCEDIMIENTO:}

*. Se comenta que la actividad se realizará de manera individual.

* Después se les colocará en la banca una hoja que tiene una figura geométrica, (el adulto que reparta las hojas deberá colocarlas de tal manera que la figura geométrica este en diferentes posiciones -arriba, abajo, a la derecha o a la izquierda-).

* Se indica que no pueden mover la hoja y se solicita que los alumnos respondan a la pregunta ¿qué es y dónde está? a través del dibujo.

* Posteriormente se les menciona pueden emplear lápiz, colores, crayolas para que realicen el dibujo de lo que creen que se trata.

* Cuando terminen cada alumno pasará al frente y mostrará su dibujo para que los otros compañeros adivinen lo que se dibujó.

- La hoja estímulo fue retomada de Modelo para la Estimulación del Pensamiento Creativo (MEPC) de Duarte (2003). 


\section{Ejemplo de actividad de enriquecimientoII \\ (Creatividad Verbal)}

\section{ACERTIJO DEL TULIPAN}

\section{OBJETIVO:}

帏 Fomentar la fluidez, elaboración y la originalidad en el área verbal, a través de encontrar la solución de un acertijo.

\section{PROCEDIMIENTO:}

登 Se indica que se trabajará de manera individual y que se tratara de adivinar un acertijo.

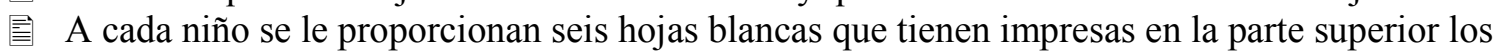
números del 1 al 5 y en la sexta hoja esta escrita la palabra respuesta.

咅 En una bolsa existen cinco pistas escritas que les ayudaran a identificar un objeto, animal o cosa y en un sobre esta la respuesta correcta al acertijo.

重 Se solicita que cada uno de los niños tome una pista y que no la lean hasta que se les indique.

童 Después se solicita que el alumno que tenga la pista número uno la lea a sus compañeros y que escriban en la hoja con el mismo número las ideas que tengan para resolver el acertijo. De esta forma se continuarán leyendo las cuatro pistas restantes hasta que se adivine el acertijo.

童 Al terminar cada alumno menciona la respuesta que encontró al acertijo y se leerá la respuesta que se encuentra en el sobre.

立 Papeletas con las pistas del acertijo del Tulipán*:

Pista1: Es una flor holandesa.

Pista 2: Su nombre significa turbante y surgió por la forma de la flor.

Pista 3: La planta en realidad se llama Nayuat Hydrongea.

Pista 4: Fue importada de Japón.

Pista 5: Es muy colorida y al mismo tiempo pálida.

* El acertijo fue elaborado por un alumno que participo en el programa. 